\title{
JUSTIFICACIÓN DEL ROL DE LAS CORTES HACIENDO JUSTICIABLES LOS DERECHOS ECONÓMICOS, SOCIALES Y CULTURALES, EN PARTICULAR, EL DERECHO A LA PROTECCIÓN DE LA SALUD
}

\author{
ARGUMENTS TO DEFEND THE ROLE OF COURTS IN THE \\ JUSTICIABILITY OF ECONOMIC, SOCIAL AND CULTURAL RIGHTS, \\ SPECIFICALLY, THE GUARANTEE OF HEALTH WELFARE
}

\section{Rodolfo Figueroa García-Huidobro*}

\begin{abstract}
RESUMEN: Existen básicamente dos objeciones en contra de que las cortes hagan justiciables los derechos económicos, sociales y culturales (DESC). Según la objeción de incapacidad, los jueces no están institucionalmente equipados para conocer los complejos y policéntricos asuntos que están envueltos en los DESC. Por ende, serían incapaces de hacer justiciables los DESC. Conforme la objeción de legitimidad, los DESC se plasman a través de políticas públicas impulsadas por el Estado. Por eso, controlar los DESC implica controlar las políticas públicas, lo que vulnera el principio de separación de poderes. Eso es ilegítimo. Ambas objeciones han sido analizadas y discutidas en la literatura angloamericana y la conclusión es que ninguna de las dos constituye un obstáculo insalvable para impedir que los jueces puedan hacer exigibles los DESC. Por el contrario, existen poderosas razones para favorecer ese rol de los tribunales.
\end{abstract}

Palabras clave: justiciabilidad, control de políticas públicas, derechos económicos, sociales y culturales.

ABSTRACT: There are basically two objections against courts making economic, social and cultural rights justiciable. According to the objection of incapacity, judges are not institutionally equipped to recognize complex and polycentric issues embedded in economic and social rights. Therefore, they would be incapable of making those rights justiciable. According to the objection of illegitimacy, economic and social rights are realized through public policies fostered throughout the Executive Branch. Consequently, judiciary control over economic, social and cultural rights would lead to breaking the separation of powers. That is illegitimate. Both objections have been analyzed and discussed in the Anglo-American literature and the conclusion is that none of them represent an insurmountable obstacle to regard economic, social and cultural rights recognizable and judged by courts. Otherwise, there are powerful reasons for promoting this labour.

Key words: Justiciability; control of public policies; economic, social and cultural rights.

* Licenciado en Ciencias Jurídicas y Sociales Universidad Diego Portales, LL.M. y S.J.D. Universidad de Wisconsin, Profesor de Derecho Constitucional de la Universidad Diego Portales. Correo electrónico: rodolfo.figueroa@udp.cl 


\section{INTRODUCCIÓN}

Este artículo tiene por objeto presentar y explicar las diferencias entre los denominados derechos civiles y políticos (DCP) y los derechos económicos, sociales y culturales (DESC), que es posible hallar en la literatura de habla inglesa, y someter tales diferencias a análisis crítico. La razón para abordar esta empresa está vinculada a la justiciabilidad de los DESC. Algunos sostienen que los DESC no son justiciables porque presentan ciertas particularidades que los distinguen de los DCP, que sí son justiciables. Así pues, la justiciabilidad o no justiciabilidad de los DESC está vinculada a las diferencias que habría entre ellos y los DCP. La intención del trabajo es demostrar que tales diferencias no justifican la pretensión de no justiciabilidad de los DESC. En la primera sección se explicarán esas diferencias y en la segunda se someterán a crítica.

\section{DIFERENCIAS ENTRE LOS DERECHOS CIVILES Y POLÍTICOS Y LOS DERECHOS ECONÓMICOS, SOCIALES Y CULTURALES}

Para tener una visión panorámica, podemos partir con la exposición de Scott y Macklem (1992, p. 24): "los derechos sociales son vistos como positivos y, por ello, involucran acción de parte del gobierno; son intensos en cuanto al uso de recursos y, por ello, son caros de proteger; son progresivos y, por ello, requieren tiempo para ser implementados; son vagos en términos de las obligaciones que imponen, y finalmente, implican complejos, policéntricos y difusos intereses en los bienes colectivos"1. A continuación, se explicarán las diferencias y sus argumentos.

\section{Derechos Positivos/Derechos Negativos; InTERVEnCión DeL Estado/Abstención del Estado; Derechos COStosos/Derechos SIN COSTO \\ Estos tres pares de criterios se colocan juntos en el título porque comparten la} misma lógica, aunque en la exposición subsiguiente se separarán en tres:

\subsection{Derechos positivos-negativos}

Algunos autores (Kelley, 1998, p. 23; Cranston, 1967, pp. 50-51; sin adherir, Eide, 1989 , p. 36) creen que existen diferencias de naturaleza entre los DCP y los DESC. Los DCP son derechos negativos mientras los DESC son derechos positivos: " $[\mathrm{u}] \mathrm{n}$ derecho positivo es un reclamo de algo -como un bien, un servicio- mientras que un derecho negativo es un derecho a que algo no sea hecho, que alguna imposición no se ejecute". (Fried, 1978, p. 110) ${ }^{2}$. Algunos autores (De Villiers, 1995, p. 602-603; Alston, 19811982, p. 310) llaman a los DCP derechos atributivos o derechos de libertad, y serían derechos de primera generación, en tanto los DESC son créditos contra el Estado y correspon-

\footnotetext{
1 Traducción libre del autor.

2 Traducción libre del autor.
} 
derían a derechos de segunda generación ${ }^{3}$. Derechos clásicos son las libertades de acción (derechos de libertad) en tanto los derechos de bienestar son derechos a ciertos bienes y equivalen a necesidades. La diferencia puede expresarse como freedom from y freedom to, thou shall not y thou shalt (Hunt, 1996, p. 45) y es una diferencia de contenido. Así, los derechos de libertad clásicos implican procesos mientras que los derechos de bienestar implican resultados (Kelley, 1998, pp. 22-24). Los derechos de libertad determinan la manera en que los individuos pueden interactuar, pero no garantizan el logro de resultados. Hablando en el medio angloamericano, Kelley señala que "[l]a Declaración de Independencia otorga a las personas el derecho a perseguir la felicidad, pero no el derecho a la felicidad per se. Los derechos de bienestar, por el contrario, intentan asegurar el éxito, al menos a nivel mínimo. Son concebidos como titularidades para gozar de ciertos bienes, no solo como el derecho a la persecución de los mismos. Son derechos a tener bienes proporcionados por otros cuando uno no puede obtenerlos por sí solo" ${ }^{4}$. Kelley recuerda que un derecho siempre impone sobre otro individuo una obligación correlativa. En el caso de derechos de libertad, lo único que imponen sobre otros es la obligación de no interferir, pero no fuerzan a nadie a actuar contra su voluntad, como es la concepción clásica de los derechos. Diferente es la situación -concluye este autor- de los derechos positivos, que imponen sobre otros obligaciones positivas que ellos no consintieron y que no pueden ser relacionadas con ningún acto voluntario.

Relacionado con el eje positivo-negativo, algunos autores presentan otro ángulo: la distinción entre acciones y omisiones. Esta sería una distinción lógica y una diferencia categorial entre abstenerse de matar y disponer de los medios para preservar la vida, entre no matar y no prestar los recursos para mantener la vida. (Hill, 1992, pp. 3-4,

\footnotetext{
3 De Villiers identifica las tres generaciones de derechos: "First-generation rights refer to the traditional liberal civil and political rights which in turn are divided into procedural and substantive rights. The following are examples of procedural rights: protection against arbitrary arrest; protection against detention without trial; the right to a public trial, to information regarding charges, to legal assistance and to be tried in a language you understand. The substantive rights include the right to be treated equally and the freedom of religion, expression, assembly, association, and movement. Second-generation rights refer especially to various aspects of social, cultural, and economic rights, such as the rights to employment, social security, shelter, family assistance, education, mother-tongue education, and state support of cultural activities. Third-generation rights refer especially to the right to self-determination, peace, development, and a protected environment". (DE VILLIERS, 1995, pp. 602-603). Fuera de ello, el autor señala que la conceptualización de los derechos como libertades negativas puede rastrearse en Locke. "The core of the Lockean philosophy regarding the protection of fundamental rights can be summarized as follows: The individual is legally entitled to an environment that is free of undue state interference. Any unlawful acts committed by the state that encroach on the rights of individuals can be nullified by a competent court of law. The rights of individuals are defined in negative terms, in that they can prevent the state interference in the lives of individuals, while individuals cannot legally require the state to assist them in the realization of their rights or the improvement of their social and economic welfare. *The state has the duty towards individuals to withhold itself from any unlawful action that may encroach upon rights of individuals. The state is under no legal obligation (in contrast to the moral and political obligations that it may have) to take positive action in support of individuals regarding their social and economic situation. The rights of individuals in terms of the Lockean approach are generally referred to as "first generation" rights in that they define the area of individual exclusivity where state action is prohibited. The state has no legal duty to improve the economic, social, and welfare position of individuals".

${ }^{4}$ Traducción libre del autor.
} 
explicando sin adherir). Continúa señalando este autor que las personas tienen un derecho a que no las maten, pero no tiene un derecho a los recursos. Los individuos pueden apelar a la generosidad y al altruismo de otros, pero no tienen un derecho sobre otros. No facilitar los medios puede constituir ausencia de benevolencia pero no una infracción a un derecho; un derecho es algo exigible, la benevolencia no.

\subsection{Abstención-intervención.}

El referido eje positivo-negativo conduce a la idea de abstención e intervención de parte del Estado. Los DCP son negativos en tanto los DESC son positivos; los DCP requieren no interferencia del Estado, mientras que los DESC sí la requieren (explicando sin adherir, Hunt, 1993, p. 149; Alston et al., 1987, p. 159; Vierdag, 1978, p. 80; Plant, 2003, p. 8, Koch, 2002, p. 31, y Abramovich et al., 2002, pp. 21-22); necesitan una activa participación del gobierno, sin la cual no pueden hacerse realidad (Alston, et al., 1987, p. 159). Los derechos de libertad difieren de los derechos de bienestar en relación con su estructura legal y los programas gubernamentales que se requieren para su implementación. Los primeros requieren un aparato legal pequeño, "the night watchman state", mientras los segundos exigen un extenso aparato estatal con elevados costos para el Estado (Kelley, 1998, p. 26).

Esta distinción entre derechos positivos de intervención y derechos negativos de abstención es central para quienes intentan presentar un argumento en contra de la revisión judicial de los DESC ${ }^{5}$. Esa tesis sostiene que los derechos negativos pueden ser protegidos mediante una decisión que invalide o revoque un acto que infrinja el derecho, emanada de las cortes. Sin embargo, un derecho positivo requiere que algo sea hecho, y para implementarlo existen múltiples opciones, dependientes de una evaluación política. Cuando la autoridad competente adopta su decisión, resulta difícil pensar en la intervención de un tribunal porque pondría a los jueces a elegir entre alternativas políticas, algo para lo cual carecen tanto de legitimidad como de experticia (explicando sin adherir, Mureinik, 1992, p. 468) ${ }^{6}$.

\footnotetext{
5 Refiriéndose a los DESC, Koch señala: "This "dependance" on resources is consequently often seen as a hindrance to the acceptance of social rights as justiciable rights. Some argue that such an acceptance would imply a limitation of the freedom of the legislature to pursue an appropriate economic "politicy" (KOCH, 2002, p. 31).

${ }^{6}$ Cranston presenta esa misma tesis y se refiere a este tema desde la perspectiva de los derechos humanos. En su opinión, solo los tradicionales DCP son derechos humanos, como el derecho a la vida, a la libertad o el debido proceso. En relación con los DESC, él sostiene que han sido puestos en una categoría equivocada; ellos no son derechos humanos, por dos razones, que él denomina la objeción filosófica y la objeción política. Según la primera, "la nueva teoría de los derechos humanos no hace sentido, y según la segunda, la circulación de una noción confusa de derechos humanos menoscaba la protección efectiva de los que sí son derechos humanos". (CRANSTON, 1967, p. 43). Cranston cree que para que algo pueda ser considerado un derecho humano es necesario que pase ciertos tests de autenticidad: practicabilidad, genuina universalidad e importancia capital (pp. 50-51) "[l]os tradicionales derechos civiles y políticos se pueden fácilmente asegurar mediante la legislación. Dado que tales derechos en su mayoría son derechos en contra de la interferencia del gobierno en las actividades de las personas, buena parte de la legislación necesaria no requiere más que restringir el brazo ejecutivo del gobierno. Este no es, sin embargo, el caso cuando pensamos en el derecho al trabajo o el derecho a la seguridad social, entre otros. Para proporcionar seguridad social se requiere más que instituir leyes; es necesario disponer de gran riqueza, pero muchos gobiernos en el mundo hoy son pobres". (pp. 50-51). Traducción libre del autor.
} 
Según Kelley (1998, pp. 26-27) la implementación de los derechos de libertad requiere que el gobierno brinde protección contra interferencias de terceros, estableciendo un Código Penal que castigue los delitos; un Código Civil y tribunales que resuelvan disputas privadas, y regulaciones constitucionales que impidan al gobierno infringir los derechos de los ciudadanos. Agrega este autor que estas normas son relativamente simples, básicamente prohíben ciertos tipos de acciones y no requieren un gran aparato estatal; el único gasto necesario es militar, para proteger frente a agresiones. En cambio, la implementación de los derechos de bienestar requiere mucho mayor activismo de parte del gobierno; involucra programas asistenciales a gran escala, mediante los cuales se transfiere riqueza, vía impuestos, hacia aquellos a quienes el Estado hace titulares de ciertos bienes. El problema es que el desarrollo económico y tecnológico de la sociedad afecta el grado en el cual ella pueda proveer derechos de bienestar a sus miembros. Incluso en una sociedad rica -sostiene Kelley- la demanda potencial por bienes -como salud- carece de límites. El proceso político decide sobre el acceso a esos bienes, y determina qué constituye el mínimo (necesidad) y qué es lujo, aunque no existe un estándar universal y no arbitrario para hacerlo y, por ende, para definir el contenido de los derechos de bienestar. Ese contenido depende del nivel de riqueza de una sociedad determinada.

\subsection{Derechos costosos-sin costo.}

Este criterio depende directamente de los criterios anteriores. Los derechos negativos no requieren intervención de parte del Estado y son, por ello, derechos sin costo. Por el contrario, los derechos positivos demandan intervención del Estado, la que siempre es muy costosa (explicando sin adherir, Plant, 2003, p. 8). Es por esto que los derechos económicos solo pueden ser implementados de acuerdo con las capacidades económicas de cada Estado y, por la misma razón, ellos no pueden ser considerados como universales. Costos excesivos sería un argumento en contra de la exigibilidad de los DESC.

Este argumento presupuestario apunta también al rol que le cabe a la judicatura en relación con la justiciabilidad de los DESC. Dado que estos derechos demandan una gran cantidad de recursos, los jueces no pueden adoptar decisiones concernientes al gasto fiscal. Mureinik (1992, pp. 465-466) explica este argumento señalando que la exigibilidad o protección de los DESC obviamente cuesta gran cantidad de dinero, pero los jueces no deben decidir sobre cómo gastar los recursos. Los jueces no disponen de un presupuesto. Además, no están calificados para evaluar cuánto se debe gastar de los recursos públicos ni cómo la sociedad puede afrontar esos gastos; tampoco deben decidir cuáles deben ser las prioridades sociales. Continúa este autor señalando que, según este argumento, estas preguntas y problemas deben estar a cargo de una autoridad que tenga tanto experticia como responsabilidad política. En una sociedad bien ordenada -sostiene Mureinik- los legisladores tienen responsabilidad política, los técnicos poseen conocimiento experto y el gobierno decide el balance entre expertizaje y política, pero los jueces no son ni electos ni expertos y por ello están mal posicionados para efectos de responsabilidad política o juicio experto. 


\section{OBLIGACIONES PRECISAS VERSUS OBLIGACIONES IMPRECISAS Y POLÍTICAS}

Este es el segundo criterio que es posible hallar en alguna doctrina para distinguir DCP y DESC. Explicaré esta distinción, separando la vaguedad del carácter político.

\subsection{Vaguedad}

Algunos autores (Alston et al., 1987, p. 159; Alston, 1997, p. 189; Plant, 2003, p. 8, Eide, 1989, p. 35) explican que las obligaciones adjuntas a los DCP pueden ser consideradas precisas mientras aquellas otras vinculadas a los DESC deben ser estimadas como imprecisas y carentes de una definición bien ajustada ${ }^{7}$. Melish (2002, pp. 33-34) explica que la naturaleza y extensión de las obligaciones que tienen los Estados, bajo el derecho internacional, provenientes de los DESC, poseen un alto grado de imprecisión. Normas que señalan que los individuos poseen derecho a comida o a vivienda adecuada, no proporcionan orientación acerca de su contenido normativo, ámbito e implicancias legales. Esto sería un problema, según autora, porque la precisión terminológica es esencial para asegurar los derechos en un contexto jurídico. La falta de una clara y explícita elaboración relativa al ámbito, contenido y las obligaciones de las partes, tiene como consecuencia que ese derecho es legalmente inoperativo y no puede ser demandado ante una corte. De Villiers (1995, pp. 606-607) recuerda que la vaguedad y ambigüedad de estos derechos existe no solo entre los diversos países sino al interior de cualquier país dado, y su imprecisión impide que tales derechos puedan satisfacer los requerimientos necesarios de justiciabilidad, tales como predictibilidad, importancia capital y universalidad. Finalmente, se pregunta cuáles son los límites para las obligaciones que provienen de los DESC. Se ha apuntado que las obligaciones de satisfacer o cumplir en materias económicas son infinitas; los DESC -sostiene este argumento- carecerían de límites.

\subsection{Carácter político 8}

Un autor (Hunt, 1993, p. 153) llama a esta la objeción politica, aunque no adhiere a ella: la implementación de los DESC involucraría decisiones políticas de gran enverga-

\footnotetext{
${ }^{7}$ ALSTON (1997) p. 189, apunta: "la principal respuesta que me dan cuando pregunto por qué usted no hace nada por tales derechos es: no sabemos cómo hacerlo. Prácticamente todos los involucrados -funcionarios públicos, políticos, abogados, defensores de los derechos, etc.- van a decir que encuentran que los estándares para los DESC son imprecisos y poco claros; que las obligaciones que recaen sobre los gobiernos son vagas en términos excesivos; que la complejidad introducida por la limitación en los recursos disponibles es demasiado grande como para incorporarla como variable en la ecuación de los derechos humanos; y para rematar, que la ausencia de un consenso profundo tanto doméstico como internacional acerca de tales derechos significa simplemente que no existe la voluntad para cambiar la situación". (Adaptación con traducción libre del autor). EIDE (1989) p. 35, explica la situación en términos similares: "[t]anto el contenido preciso de una serie de derechos económicos, sociales y culturales, así como las obligaciones específicas que imponen para los Estados parte del Pacto Intenacional de Derechos Económicos, Sociales y Culturales, permanece extremadamente vaga. Esta vaguedad, cuando es contrastada con la precisión con que la mayoría de los derechos civiles y políticos han sido elaborados, ha tendido a incentivar una relativa negligencia por los derechos económicos y sociales" (traducción libre del autor).

${ }^{8}$ Debo aclarar que para efectos de claridad, expondré en este texto esta objeción, pero no será contestada porque se ha dedicado un artículo exclusivamente a este asunto, titulado "El rol de las cortes haciendo justiciables los derechos económicos, sociales y culturales, en particular, el derecho a la protección de la salud”, en la Revista Chilena de Derecho, Vol. 36 No 2, 2009.
} 
dura, comparada con el caso de los DCP. Estos asuntos deben ser dejados a la decisión de legisladores, ministros y otros actores políticos. Los jueces pueden apropiadamente resolver casos que involucren los DCP pero no los DESC, cuya implementación -por ser una cuestión política- no un asunto del derecho y no es un tema de derechos (Vierdag, 1978, p. 103). Los deberes que emanan de estos derechos deben ser dejados para la negociación política, no estar completamente definidos, como lo están en el caso de los derechos negativos. La indeterminación del derecho y sus deberes tiene como efecto un proceso constante de disputa, negociación y adjudicación, a diferencia de lo que ocurre con los derechos negativo (explicando sin adherir, Plant, 2003, p. 8). Por eso, los jueces no deben participar en estas deliberaciones, porque carecen de dos cualificaciones esenciales: experticia y responsabilidad política (sin adherir, Mureinik, 1992, p. 465). "Las cortes carecen de las herramientas de la burocracia; no pueden crear programas gubernamentales; no tienen una visión sistemática de las políticas del gobierno. En estas circunstancias, no es realista esperar que las cortes hagan exigibles los derechos positivos" (Sunstein, 1993, p. 37. Explicando sin adherir, Brems, 2001, p. 447; Jackman, 1992, p. 18; Abramovich et al., 2002, p. 42); no están institucionalmente equipados para manejar y resolver las complejidades que envuelve el policy making process. Puestos los jueces a hacer esto, probablemente adoptarán malas decisiones políticas, además de infringir el principio de separación de poderes. Este principio impide que las cortes le señalen al parlamento cómo debe decidir acerca del gasto de los recursos públicos (explicando sin adherir, De Villiers, 1995, pp. 606-607; Melish, 2002, p. 33).

\section{EFECTIVIDAD INMEDIATA/PROGRESIVIDAD}

Este es un tercer criterio para distinguir los DESC de los DCP. El argumento es que los DCP pueden ser demandados de inmediato del Estado porque implican obligaciones negativas. En cambio, la situación de los DESC sería diferente porque ellos requieren de la intervención del Estado, cumpliendo obligaciones positivas, las que solo pueden ser satisfechas de manera progresiva. "Los derechos civiles pueden ser asegurados del mismo modo en todos los países, ricos y pobres, en tanto la implementación de los derechos sociales necesariamente varía, dependiendo de la prosperidad de cada país, así como de las prioridades asignadas a esos objetivos" (Vierdag, 1978, pp. 81-82)9 . Los DCP están determinados y, por ello, son absolutos; los DESC son, por su propia naturaleza, relativos, lo que quiere decir que dependen de los métodos disponibles para su implementación. En el caso de los DCP no existe ninguna razón por la cual no puedan ser asegurados en su totalidad, todos al mismo tiempo, mientras que los DESC usualmente se implementarán de a poco, uno por uno. Por otra parte, el disfrute de los DCP puede y debe ser universal, mientras que en el caso de los DESC eso es selectivo (Vierdag, 1978, p. 82).

\footnotetext{
9 Traducción libre del autor. BEETHAM (1995) p. 42 explica esta visión: un derecho humano debe satisfacer varias condiciones: debe ser fundamental y universal; en principio, debe ser definido de manera que sea justiciable; debe estar claro quien tiene la obligación de asistirlo e implementarlo, y la agencia que sea responsable por él debe poseer la capacidad de cumplirlo. El problema es que los DESC no cumplen con estas condiciones.
} 


\section{OTROS ARGUMENTOS}

Existen otros argumentos y comentarios que pretenden distinguir entre los DCP y los DESC, que probablemente son de menor relevancia que los ya mencionados. Por ello, los presentaré de modo breve.

\subsection{Base ideológica}

En parte relacionado con el primer criterio, se ha argüido que los DCP son no ideológicos en su naturaleza y son potencialmente compatibles prácticamente con cualquier régimen político. Por el contrario, los DESC son a menudo percibidos como profundamente ideológicos, requiriendo una inaceptable intervención del Estado en asuntos domésticos; ellos son inherentemente incompatibles con una economía de libre mercado (explicando sin adherir, Alston, et al., p. 1987, p. 160).

\subsection{Falta de compromiso}

Los DCP están relacionados con valores ampliamente compartidos, el tipo de valores con los cuales los gobiernos están en verdad comprometidos. Esos derechos generan problemas que los gobiernos sí pueden manejar. En cambio, los DESC aún no han logrado alcanzar un genuino compromiso de parte de los gobiernos y representan problemas que, en general, son inmanejables o difícilmente manejables (Alston, et al., p. 1987, pp. 160-161, 189). La ausencia de un profundo compromiso con ellos, tanto a nivel doméstico como internacional, significa simplemente que no existe voluntad para el cambio.

\subsection{Autonomía vs. Incapacidad}

Kelley (1998, pp. 28-29) ha presentado un argumento filosófico en contra de los DESC, basado en una cierta concepción de los seres humanos. Los derechos de libertad o derechos clásicos están relacionados con el Enlightenment individualism, mientras que los derechos de bienestar pertenecen a cierto tipo de colectivismo. Los derechos de libertad dejan que los individuos sean responsables de vivir sus propias vidas, satisfacer sus propias necesidades, perseguir sus propios fines, utilizar y disponer de los recursos materiales que hayan adquirido producto de su esfuerzo, y garantizan la libertad necesaria para asumir esas responsabilidades. Esos derechos reflejan la suposición de que los individuos son fines en sí mismos, que no deben ser empleados en contra de sí mismos para alcanzar propósitos sociales. Los derechos de bienestar, en cambio, están basados en una concepción de los individuos como "inextricablemente enraizados en sus comunidades"; asignan titularidades sobre los recursos de la comunidad. "[L]os derechos de libertad reflejan una filosofía política individualista que premia la libertad, en tanto los derechos de bienestar adhieren a una visión comunitaria o colectivista que está dispuesta a sacrificar la libertad" ${ }^{10}$.

\footnotetext{
10 Traducción libre del autor. Por su parte, PLANT (2003) p. 3, también explica este argumento señalando que los críticos de los derechos sociales distinguen entre libertad negativa y libertad positiva y defienden la idea de que solo la libertad negativa debe ser protegida por los derechos. La ausencia de coerción (libertad negativa) debe ser distinguida de la habilidad o capacidad (libertad positiva). Los DESC no deben ser considerados genuinos derechos sino solo "condiciones contingentes discutibles para el ejercicio de genuinos derechos. Ellos son más bien materia de políticas públicas que derechos constitucionales". (PLANT, 2003, p. 3). Traducción libre del autor.
} 


\subsection{Pobreza}

Otro argumento en contra de los DESC está basado en el hecho de la pobreza. Se sostiene que los DESC no son remediables porque no existe un agente causal de la pobreza (Sin endosar, Melish, 2002, p. 33). Los derechos negativos pueden ser intencionalmente quebrantados cuando las obligaciones de no interferir no son respetadas, pero con los derechos positivos es distinto, pues ellos requieren recursos y si los recursos no están disponibles, no existe ningún acto ilícito. La injusticia solo puede ser causada por un acto intencional, pero la pobreza y la falta de recursos no corresponde a esa hipótesis. Incluso asumiendo que los recursos económicos y sociales forman parte constitutiva de la agencia humana, no es algo incorrecto ni injusto si alguien carece de los recursos necesarios para ser agente como consecuencia de un proceso impersonal y no deliberado (Plant, 2003, p. 13).

Con esta explicación concluye la sección I, destinada a presentar las diferencias entre los DCP y los DESC. Ahora pasamos a la sección II, donde tales diferencias se someten a crítica.

\section{DISCUSIÓN SOBRE LOS DIVERSOS CRITERIOS PARA DISTINGUIR DCP Y DESC}

\section{Derechos positivos/negativos; AbStención Del Estado/ INTERVENCIÓN DE ESTADO; DERECHOS COSTOSOS/SIN COSTO \\ Esta es la distinción más frecuentemente presentada para distinguir DCP y DESC} y es la más importante porque constituye la base para todas las demás. En la literatura de habla inglesa encontramos diversos comentarios sobre la referida distinción:

1.1. Probablemente la objeción más importante a esta distinción proviene de la tesis de que todo derecho comprende tres niveles de obligaciones. Esta perspectiva es conocida como el triple esquema de obligaciones, que estaría presente en todo derecho. La realización completa de cualquier derecho involucra la ejecución de múltiples tipos de deberes (Shue, 1980, p. 52, 55) lo que lleva a distinguir tres niveles de obligaciones: a) obligaciones de evitar privar un derecho; b) obligaciones de proteger de la privación, y c) obligaciones de auxiliar al privado de su derecho. Todo derecho posee estos tres niveles. El deber de evitar a privación es negativo, requiere mera abstención, en tanto los deberes de proteger y de auxiliar son positivos y requieren intervención de parte del Estado.

Eide (1989, p. 37; 1992, p. 5) reformuló este esquema de Shue quedando de la siguiente manera, que es la más difundida:

a) Obligación primaria de respetar: "[r]equiere del Estado y de todos su órganos y agentes que se abstengan de hacer nada que viole la integridad del individuo o infrinja su libertad de usar los recursos materiales disponibles para él en la forma en que considere mejor para satisfacer sus necesidades básicas" (Eide, 1992, p. 5. También, Hunt, 1996, p. 31; Puta-Chekwe et al., 2001, p. 43) ${ }^{11}$.

11 Traducción libre del autor. 
b) Obligación secundaria de proteger: "[r]equiere del Estado y sus agentes las medidas necesarias para prevenir que otros individuos o grupos violen la integridad, libertad de acción u otros derechos humanos del individuo, incluyendo impedir el disfrute de sus recursos materiales" (Eide, 1992, p. 5; Hunt, p. 32; PutaChekwe et al., p. 43) ${ }^{12}$.

c) Obligación terciaria de satisfacer ${ }^{13}$ : "[r] equiere del Estado adoptar todas las medidas necesarias para asegurar a cada persona, dentro de su jurisdicción, las oportunidades de obtener satisfacción de sus necesidades, reconocidas en los instrumentos de derechos humanos, que no puedan ser aseguradas mediante esfuerzos personales" (Eide, 1992, p. 5; Hunt, p. 37; Puta-Chekwe et al., p. 43) ${ }^{14}$.

Este esquema permite apreciar la existencia de varios niveles de obligaciones (Hunt, 1996, p. 31) en cada derecho ${ }^{15}$, tanto positivas como negativas, así como abstención e intervención de parte del Estado. Además, todo derecho es a la vez costoso y sin costo para el Estado, dependiendo del tipo de obligaciones de que se trate: las obligaciones terciarias obviamente serán más costosas que las primarias. Así las cosas, la distinción simplista en comento no tiene sentido (Abramovich, et al., 2002, p. 29). Por otra parte, este esquema muestra que la distinción positivo-negativo debe aplicarse más bien a las obligaciones que emanan de los derechos que a los derechos mismos (Shue, 1980, p. 53). Finalmente, debemos tener en cuenta que si nos interesa que los derechos sean satisfechos en su globalidad, entonces es necesario satisfacerlos en los tres niveles (Shue, 1980, p. 53; Beetham, 1995, p. 51). En efecto, no pensaríamos que nuestro derecho a la vida está seguro -señala Shue- si nos limitamos a esperar que el Estado no nos mate (obligación primaria), sin esperar, igualmente, que tampoco un tercero lo haga y que el Estado adopte medidas positivas para proteger el derecho e impedir que eso ocurra (obligación secundaria). Del mismo modo, si pensamos en un derecho civil, como el

\footnotetext{
12 Traducción libre del autor.

${ }^{13} \mathrm{El}$ original dice fulfill, que aquí traducimos como satisfacer/cumplir.

${ }^{14}$ Traducción libre del autor.

15 Si bien es cierto que el esquema es creado por Shue solo para los derechos básicos, él considera que los derechos básicos son los derechos de seguridad y los derechos de subsistencia. Por ello, si entendemos que los DESC están relacionados con los derechos de subsistencia, entonces el esquema es aplicable tanto a los DCP como a los DESC. Además, SHUE (1980, p. 52) señaló expresamente que muchos otros derechos, además de los llamados derechos básicos, tienen tres niveles de obligaciones. Cuando Eide trabajó en el esquema múltiple, en su artículo de 1992, habló de las obligaciones del Estado hacia los derechos humanos en general, tanto los DCP como los DESC: "The content of the human rights principles adopted by the United Nations is broader and more multidimensional than anything known in the past. It derives from the list of rights which together forms the human rights system: the corpus of civil, political, economic, social and cultural rights. From these can be derived at least the following principles of good governance: popular sovereignty, a domain of freedom for the individual, rule of law, and social justice. The threefold obligation of government, if these are to comply with international human rights, is as follows: to respect, to protect, and to fulfil human rights". (EIDE, 1992, p. 5). Fuera de ello, en su artículo de 1989, hablando en particular sobre la realización de los DESC, él pensó que el esquema de los tres niveles se aplica a todo derecho, no solo a los DESC. "State responsibility for human rights can be examined at three levels: The obligation to respect, the obligation to protect, and the obligation to fulfil human rights". (EIDE, 1989, p. 37).
} 
derecho a un debido proceso, veremos que las obligaciones terciarias son fundamentales (que el Estado proporcione cortes, jueces y auxiliares, procedimientos, policía, laboratorios, regulaciones de diverso tipo, etc.). Claramente, el cumplimiento solo de los niveles primario y secundario en el caso del debido proceso no basta para que el derecho se entienda cumplido.

Este esquema de obligaciones múltiples ha sido aceptado, desde hace mucho tiempo, no solo por doctrina comparada sino por jurisprudencia internacional ${ }^{16}$. Además, el Comité de Naciones Unidas sobre los DESC adoptó este esquema, declarando que es aplicable a todo derecho humano ${ }^{17}$. Las Directrices de Maastricht también recogen este esquema tripartito, en la $\mathrm{N}^{0} 6^{18}$.

En suma: si todo derecho comprende obligaciones positivas y negativas, entonces todo derecho es positivo y negativo a la vez, requiere tanto abstención como interven-

16 Por ejemplo, en el caso Velásquez Rodríguez de la Corte Interamericana de Justicia, y en el caso X e Y v. The Netherlands, de la Corte Europea de Derechos Humanos, que no son casos de DESC. En el caso Airey, la Corte Europea cuestiona la distinción entre libertades clásicas y derechos sociales. (PELLONPÄÄ, 1993, p. 859).

17 Así, los tres niveles aparecen incluidos en el Comentario General № 12, sobre el derecho a comida adecuada: "The right to adequate food, like any other human right, imposes three types or levels of obligations on States parties: the obligations to respect, to protect and to fulfil. In turn, the obligation to fulfil incorporates both an obligation to facilitate and an obligation to provide. The obligation to respect existing access to adequate food requires States parties not to take any measures that result in preventing such access. The obligation to protect requires measures by the State to ensure that enterprises or individuals do not deprive individuals of their access to adequate food. The obligation to fulfil (facilitate) means the State must proactively engage in activities intended to strengthen people's access to and utilization of resources and means to ensure their livelihood, including food security"; sobre el derecho a la educación, en el Comentario General No 13: "The obligation to respect requires States parties to avoid measures that hinder or prevent the enjoyment of the right to education. The obligation to protect requires States parties to take measures that prevent third parties from interfering with the enjoyment of the right to education. The obligation to fulfil (facilitate) requires States to take positive measures that enable and assist individuals and communities to enjoy the right to education. Finally, States parties have an obligation to fulfil (provide) the right to education"; sobre el derecho al más alto nivel posible de salud, en el Comentario General No 14: "The right to health, like all human rights, imposes three types or levels of obligations on States parties: the obligations to respect, protect and fulfil. In turn, the obligation to fulfil contains obligations to facilitate, provide and promote. The obligation to respect requires States to refrain from interfering directly or indirectly with the enjoyment of the right to health. The obligation to protect requires States to take measures that prevent third parties from interfering with article 12 guarantees. Finally, the obligation to fulfil requires States to adopt appropriate legislative, administrative, budgetary, judicial, promotional and other measures towards the full realization of the right to health". Etc.

18 Maastricht Guidelines $N^{\circ}$ 6. Like civil and political rights, economic, social and cultural rights impose three different types of obligations on States: the obligations to respect, protect and fulfil. Failure to perform any one of these three obligations constitutes a violation of such rights. The obligation to respect requires States to refrain from interfering with the enjoyment of economic, social and cultural rights. Thus, the right to housing is violated if the State engages in arbitrary forced evictions. The obligation to protect requires States to prevent violations of such rights by third parties. Thus, the failure to ensure that private employers comply with basic labour standards may amount to a violation of the right to work or the right to just and favourable conditions of work. The obligation to fulfil requires States to take appropriate legislative, administrative, budgetary, judicial and other measures towards the full realization of such rights. Thus, the failure of States to provide essential primary health care to those in need may amount to a violation. 
ción de parte del Estado, e implica gastos para el mismo, con lo cual la distinción señalada pierde utilidad.

1.2. Es posible formular un segundo comentario a esta distinción sin recurrir al esquema de Shue-Eide. Tal distinción es problemática porque una serie de DCP requieren intervención de parte del Estado, lo que los haría positivos, tornando esta distinción en inútil (De Villiers, 1995, p. 604; Jackman, 1992, p. 23; Abramovich et al., 2002, p. 24; Koch, 2002, p. 32; Holmes et. al, 1999, capítulo 1; Peces-Barba, 1981, p. 290; Pellonpää, 1993, p. 858; Puta-Chekwe, 2001, p. 42). Por ejemplo, el comité de la ONU en contra de la tortura ha señalado que los Estados no cumplen con su obligación internacional de prohibición de la tortura y otros tratos inhumanos y degradantes meramente con la aprobación leyes que prohíban la tortura; se requiere mucho más: construir establecimientos para la detención de personas que satisfagan los estándares internacionales acerca de la detención, y establecer programas de entrenamiento para los policías y otros oficiales del Estado que estén a cargo de las cárceles. Pues bien, no cabe duda que hacer eso requiere la intervención del Estado -no mera abstención- y es muy caro - no es gratuito, y estamos hablando de un derecho civil- (Hunt, 1993, p. 150; Mureinik, 1992, p. 466). Lo mismo cabe decir de la promoción y protección de otros derechos DCP, como el derecho a tener elecciones libres y equitativas (Hunt, 1993, p. 151; Waldron, 1995, p. 580; Hirschl, 2000, p. 1072; Shue, 1980, pp. 37-38). Buenos ejemplos de obligaciones positivas que derivan de los DCP y que descansan sobre los Estados pueden encontrarse en los Comentarios Generales del Comité de Derechos Humanos del Pacto Internacional sobre Derechos Civiles y Políticos (PIDCP) ${ }^{19}$.

Los derechos negativos son importantes restricciones sobre elecciones y acciones. Por ello, no pueden ser gratuitos (Fried, 1978, pp. 112-113). Por ejemplo, un país pobre puede no tener recursos suficientes para instalar un sistema de financiamiento público de las campañas electorales con la finalidad de asegurar igualdad de oportunidades; o puede no tener la capacidad de garantizar un sistema de justicia criminal imparcial; algunos países pobres no tienen los recursos para garantizar que las fuerzas de policía puedan patrullar regularmente barrios peligrosos. Proyectando este argumento a un extremo, la capacidad de un Estado de asegurar los derechos civiles más básicos depende de su capacidad militar para evitar o disuadir a otros Estados de invadirlo y tiranizar la población. Por tanto, los derechos negativos no pueden ser considerados gratuitos o sin costo. En consecuencia, el eje positivo/negativo no facilita una clara distinción entre DCP y DESC (Eide, 1989, p. 36).

\footnotetext{
${ }_{19}$ Por ejemplo, el Comentario General No 3, sobre el Artículo 2 (Implementation at the national level); el Comentario General No 4, sobre el Artículo 3 (Equal right of men and women to the enjoyment of all civil and political rights); el Comentario General No 6, sobre el Artículo 6 (Right to life); el Comentario General $\mathrm{N}^{\circ} 20$, sobre el artículo 7 (Prohibition of torture, or other cruel, inhuman or degrading treatment or punishment); el Comentario General $N^{\circ} 12$, sobre el artículo 1 (Right to self-determination); el Comentario General No 17, sobre el artículo 24 (Rights of the child); el Comentario General No 18, sobre no discriminación; el Comentario General No 21, sobre el artículo 10 (Humane treatment of persons deprived of their liberty); el Comentario General No 23, sobre el artículo 27 (Rights of minorities).
} 
Plant (2003, p. 10) explica que los críticos de los derechos positivos pueden responder este argumento señalando dos cosas: a) los derechos negativos demandan recursos por como el mundo es, pero podemos imaginar el Reino de los Cielos, en el cual "todas las obligaciones de abstención respecto de los derechos negativos siempre se cumplan. En ese mundo posible, los derechos negativos no involucrarán nada que tenga que ver con recursos. Al contrario, en ese mundo posible, los derechos positivos de todos modos implicarán recursos, por su propia naturaleza. Por tanto, existe una diferencia lógica y esa diferencia lógica justificaría preservar la idea de que los derechos son negativos y que los derechos positivos no son genuinos derechos" ${ }^{20}$. b) Los recursos son, en el mejor de los casos, condiciones para los derechos negativos, pero no parte de su naturaleza lógica interna. Pero en el caso de los derechos positivos, los recursos están interna y lógicamente conectados con la naturaleza del reclamo.

Plant (2003, p. 11) presenta respuestas a estos dos contraargumentos. Para el primero, señala que si uno puede imaginar un mundo en el cual no exista escasez de motivación para respetar los derechos, entonces también debe ser posible imaginar que no exista escasez de recursos. Ambos tipos de derechos tiene en común la escasez y, por ende, no existe diferencia lógica entre ellos. Fuera de eso, si pensamos en el Jardín del Edén, debemos entender que allí no existe necesidad de derechos de ningún tipo. Los derechos "...se refieren a qué tipo de protección los individuos pueden tener en un mundo en el cual existe escasez tanto de motivación como de recursos" ${ }^{21}$. Respecto del segundo argumento, Plant sugiere que los recursos no son una condición de los derechos negativos que se pueda separar. Por el contrario, existe una conexión conceptual entre la idea de derechos y la justiciabilidad de los mismos 22 . La capacidad de hacer respetar un derecho no es una condición contingente del derecho sino parte de lo que constituye al derecho en derecho. Siendo así, los recursos son relevantes para ambos tipos de derechos, por lo cual la distinción falla.

1.3. El eje positivo/negativo condujo a la distinción entre acciones y omisiones, argumentando algunos autores que los derechos están vinculados con no hacer algo (como no matar), pero no con hacer algo (como proporcionar los recursos para mantener la vida). Hill (1992, p. 4) responde a ella: la causalidad existe independientemente de un contrato. Un individuo puede ser causalmente responsable, por su inacción, de un resultado que infrinja un derecho, independientemente de si él estaba vinculado por un

\footnotetext{
20 Traducción libre del autor.

21 Traducción libre del autor.

22 Para explicar esta idea, él se apoya en Steiner, que señaló: "This construal of rights as freedom allocations is sufficient to explain why those duties are uncontroversially seen as enforceable. For, putting the matter as broadly as possible, we can say that to prevent someone's chosen disposition of elements within his or her domain is to diminish that persons allotted freedom: specifically, it makes that person unfree to secure whatever is aimed at in that disposition. A set of rights-creating rules that lacked provision for the enforcement of those duties-that allowed, much less required, rights violations to stand unreversed-could not then consistently be described as doing what it purports to do: namely assigning that discretionary domain to that person. "No right without a remedy" as the legal maxim says". (PLANT, citando a Steiner, 2003, p. 11).
} 
contrato o no ${ }^{23}$. Tanto acciones como omisiones pueden resultar en violaciones de los derechos humanos de igual magnitud (Puta-Chekwe, 2001, p. 43).

1.4. Un cuarto comentario sobre la distinción positivo/negativo está relacionado con la idea de libertad. Algunos autores (como Kelley, 1998) apoyan la distinción positivonegativo sobre la base de que un derecho negativo corresponde a una libertad, en tanto un derecho positivo corresponde a bienes ${ }^{24}$. Sin embargo, desde un punto de vista analítico, no es exacto asociar derechos negativos con la libertad, porque la idea de libertad puede ella ser entendida tanto en sentido negativo como positivo, como Berlin se encargó de demostrar en su famoso discurso en $1958^{25}$. En consecuencia, la distinción es fallida.

1.5. Otro argumento en contra de la distinción entre derechos positivos y negativos está relacionado con la distinción entre obligaciones de conducta y obligaciones de resultado. Eide (1989, p. 38) y Andreasen et al. (1992, p. 256) señalan que las obligaciones de conducta pueden efectivamente ser definidas como un comportamiento, que el sujeto imperado debe ejecutar o abstenerse de ello. En contraste, las obligaciones de resultado se encuentran menos concernidas con la elección de una línea de acción y más con los resultados de ella. Esta distinción es importante para abordar el tema de satisfacer las obligaciones por parte del Estado. Goodwin-Gill (1984, p. 112) señala que en la práctica, las obligaciones de conducta son menos frecuentes que las de resultado, y que ellas son más frecuentes cuando una acción de parte del Estado es requerida. Por otro lado, las obligaciones de resultado -que reconocen el principio de poder elegir los medios- son más frecuentes cuando los Estados son requeridos de promover o crear ciertas situaciones o estados de cosas dentro de su sistema jurídico.

Beetham (1995, p. 49) y Eide et al. (1995, p. 39) consideran que esta distinción es relevante para abordar las probabilidades de hacer exigible el Pacto Internacional de Derechos Económicos, Sociales y Culturales (PIDESC). Las obligaciones asumidas por los Estados bajo el PIDESC parecen ser obligaciones de resultado más que de conducta, más de fines que de medios. Esto no implica que no existan obligaciones de conducta en el tratado, algo que explícitamente está reconocido por las Directrices de Maastricht, No $7^{26}$.

\footnotetext{
${ }^{23}$ Continúa este autor: "The theorists of positive rights assert that humans stand in quasi-contractual relations as it were with the rest of mankind based on both the shared capacity for rational agency and the shared passions of sympathy. Humans thus have a positive duty to act, beyond the existence of an explicit contract. The argument is essentially that if there is a commitment to negative rights then there is no reason in principle why that commitment does not similarly apply to economic and social rights". (HILL, 1992, p. 4).

${ }^{24}$ KELLEY, sección I de este trabajo.

25 BERLIN (1969).

${ }^{26}$ Maastricht Guidelines, No. 7. The obligations to respect, protect and fulfil each con-tain elements of obligation of conduct and obligation of result. The obligation of conduct requires action reasonably calculated to realize the enjoyment of a particular right. In the case of the right to health, for example, the obligation of conduct could involve the adoption and implementation of a plan of action to reduce maternal mortality. The obligation of result requires States to achieve specific targets to satisfy a detailed substan-tive standard. With respect to the right to health, for example, the obligation of result requires the reduction of maternal mortality to levels agreed at the 1994 Cairo International Conference on Population and Development and the 1995 Beijing Fourth World Conference on Women.
} 
Lo que es importante es que si el PIDESC está relacionado más con obligaciones de resultado que de conducta, entonces sí pueden ser justiciables tales obligaciones porque los resultados son susceptibles de alguna forma de control y, por ello, de alguna forma de justiciabilidad. Esta conclusión es relevante porque la distinción positivo-negativo tiene el propósito de bloquear la posibilidad de hacer justiciables los DESC.

1.6. Otro argumento en contra de la distinción en comento se focaliza en la suposición que subyace a tal distinción, y es que es posible identificar de manera fácil una obligación positiva correlativa con un derecho positivo y una obligación negativa con un derecho negativo. Shue (1984, p. 85) no lo cree; estima que es muy difícil intentar determinar, desde un punto de vista teorético, qué tipos de obligaciones corresponden a qué tipos de derechos y, finalmente, poder identificar todas las obligaciones que derivan de cualquier derecho. Esto sucede tanto con los DCP como con los DESC. La correlación entre los derechos negativos y los deberes de omisión, por una parte, y entre los derechos positivos y las obligaciones de asistencia, por la otra, no es fácil de mantener, y esos intentos son artificiales, simplistas y áridos (Shue, 1984, p. 84; Eide, 1995, p. 38). El set de obligaciones que un derecho pueda involucrar es muy diverso (Abramovich et al., 2002, p. 36).

1.7. Otra consideración se basa en la idea de la interdependencia y permeabilidad entre los derechos. Existe abundante literatura que explica las relaciones de interdependencia y permeabilidad que existen entre los DCP y los DESC (Addo, 1988, p. 1425; Türk, 1992, p. 19). La permeabilidad es entendida en el sentido de que los DCP son utilizados como un vehículo para la protección de los DESC (Craven, 1993, p. 400). Por ejemplo, la permeabilidad de los DESC mediante el derecho civil a la libertad personal, o el estatuto de la detención del artículo 5 de la Convención Europea (Koch, 2002, pp. 29-30). Sobre esa base, Gómez (1995, p. 155) piensa que los DCP y los DESC deben ser protegidos y promovidos con la misma intensidad y Scott (1989, p. 771) ha sostenido que las normas de derechos humanos del PIDESC pueden permear las normas del PIDCP, permitiendo que los DESC puedan ser sometidos a la jurisdicción de supervisión del Comité de Derechos Humanos bajo el PIDCP. De esa forma, los individuos podrían obtener acceso a un procedimiento que no se encuentra disponible bajo el PIDESC. Además, los derechos son interdependientes (Koch, 2002, pp. 29-30), como reconoce la Vienna Declaration and Programme of Action, de 1993: "All human rights are universal, indivisible and interdependent and interrelated"27. Estas relaciones fueron reconocidas en 1952, con la resolución No 543 y está implícita en la Convención Europea de Derechos Humanos del año 1950. Junto con ello, otros documentos de

\footnotetext{
${ }^{27}$ La sección 5 continúa señalando: "The international community must treat human rights globally in a fair and equal manner, on the same footing, and with the same emphasis. While the significance of national and regional particularities and various historical, cultural and religious backgrounds must be borne in mind, it is the duty of States, regardless of their political, economic and cultural systems, to promote and protect all human rights and fundamental freedoms".
} 
Naciones Unidas deben ser mencionados: Los Principios de $\operatorname{Limburg}^{28}$ y las Directrices de Maastricht ${ }^{29}$.

Esta es la razón por la cual algunos autores (Andreassen et al., 1992, p. 253), piensan que los DCP y los DESC están en igual pie. Brems (2001, p. 444) sostiene que "en el discurso oficial de los Estados así como en las organizaciones internacionales, existe consenso respecto de los principios de indivisibilidad e interdependencia de todos los derechos humanos: no puede haber jerarquía, todos tienen el mismo estatus" 30 .

1.8. Las consideraciones anteriores deberían reducir el entusiasmo por la distinción positivo-negativo, abstención-intervención, costosos-sin costo, aplicada a los DCP y los DESC. Algunos autores (Brems, 2001, p. 444; Shue, 1984, p. 84; Abramovich, 2002, pp. 24-25) concluyen que las diferencias entre esas dos categorías de derechos no corresponden a una diferencia cualitativa sino de grado porque ambos tipos de derechos implican obligaciones positivas y negativas. Sobre esa base, más que distinguir entre tipos de derechos, se ha sugerido que se podría distinguir entre tipos de obligaciones (Scott, 1989, pp. 834-835). Por eso, Türk (1992, p. 26) estima que las distinciones bajo análisis corresponden más bien a una distinción ideológica que a una conceptual o teorética. Keller (2002, p. 600) piensa que la "categorización de los derechos económicos como derechos positivos ilegítimos, en contraste con los derechos negativos de la Constitución, crea una dicotomía falsa" ${ }^{31}$. Probablemente la única diferencia indisputable entre DCP y DESC es que los últimos carecen de la tradición legal que tienen los primeros (Hunt, 1996, p. 69; Hunt, 1993, p. 153; Van Hoof, 1984, pp. 97-98).

\footnotetext{
${ }^{28} N^{\circ} 1$. Economic, social and cultural rights are an integral part of international human rights law. They are the subject of specific treaty obligations in various international instruments, notably the International Covenant on Economic, Social and Cultural Rights.

$N^{\circ}$ 3. As human rights and fundamental freedoms are indivisible and interdependent, equal attention and urgent consideration should be given to the implementation, promotion and protection of both civil and political, and economic, social and cultural rights.

${ }^{29}$ No. 4. It is now undisputed that all human rights are indivisible, interdependent, interrelated and of equal importance for human dignity. Therefore, states are as responsible for violations of economic, social and cultural rights as they are for violations of civil and political rights.

$N^{\circ}$ 5. As in the case of civil and political rights, the failure by a State Party to comply with a treaty obligation concerning economic, social and cultural rights is, under international law, a violation of that treaty. -Building upon the Limburg Principles, the considerations below relate primarily to the International Covenant on Economic, Social and Cultural Rights (hereinafter "the Covenant"). They are equally relevant, however, to the interpretation and application of other norms of international and domestic law in the field of economic, social and cultural rights.

30 Traducción libre del autor.

${ }^{31}$ Traducción libre del autor. Craven lo señala en estos términos: [t]here is nothing innate in the nature of economic, social and cultural rights themselves that should categorically distinguish them form traditional civil and political rights. The differences that do arise, relate to the specific State obligations that accompany such rights in the relevant international instruments. Even in the context of obligations, moreover, there has been a tendency either to exaggerate or underplay the precise situation with the effect of preventing a clear and uncontroversial picture from developing". (CRAVEN, 1993, pp. 368-369).
} 


\section{OBLIGACIONES PRECISAS VERSUS OBLIGACIONES IMPRECISAS}

Este fue el segundo criterio explicado en la primera sección para distinguir DCP y DESC. Según ese criterio, los DESC son vagos, comparados con los DCP que son precisos. Además, los DESC implican tomar definiciones en materias políticas, colocando a los jueces en una posición que no les corresponde. En lo que sigue, se presentarán objeciones al tema de la vaguedad, pero no al carácter político de los DESC y ni sobre el rol que los jueces desempeñarían adjudicando DESC ${ }^{32}$.

2.1. El principal argumento en contra de la objeción de vaguedad proviene del triple esquema de obligaciones de Shue $(1980,1984)$ y Eide $(1989,1992)$. La obligación primaria de respetar es ciertamente muy clara; la conducta que esa obligación requiere es muy precisa: abstenerse de privar a un individuo de sus derechos. En cambio, las obligaciones secundarias y terciarias de proteger y satisfacer/cumplir son imprecisas. En efecto, no siempre es claro saber qué es lo que se debe hacer para prevenir que terceros infrinjan los derechos de las personas (obligaciones secundarias) y, ciertamente, no es para nada claro qué es lo debido en orden a satisfacer/cumplir un derecho (obligaciones terciarias) (Hunt, 1996, p. 34). Lo que el esquema de triple obligación muestra es que la claridad o vaguedad de las obligaciones es algo que depende del tipo de obligación involucrada, no del tipo de derecho de que se trate, puesto que todos los derechos comprenden los mismos tres niveles de obligaciones. Siendo ese el caso, los DESC poseen obligaciones primarias que son bastante precisas, al igual que los DCP; y presentan también obligaciones secundarias y terciarias que son imprecisas, tal como sucede con los DCP en esos mismos niveles.

Según Scott y Macklem (1992, p. 76) cuando los defensores de una distinción tajante entre DCP y DESC alegan que los segundos son vagos, en verdad, están comparando los DESC a nivel terciario con los DCP a nivel primario. Pero comparar ambos tipos de derechos en distintos niveles es arbitrario, tal como lo sería comparar manzanas y naranjas ${ }^{33}$; deben compararse las obligaciones de distintos derechos en el mismo nivel (Hunt, 1996, p. 34). El error de efectuar tal comparación y, especialmente las consecuencias que se siguen de ello, se pone de manifiesto si la invertimos. ¿Qué ocurriría si comparamos DCP a nivel terciario con DESC a nivel primario? Eso nos llevaría a estimar que los DESC son muy precisos en tanto los DCP serían imprecisos. Más aún, nos llevaría a la conclusión de que los DESC son justiciables mientras que los DCP no, por ser excesivamente imprecisos. Esto es, obviamente, un error, y por eso la distinción es equivocada ${ }^{34}$. Simples ejemplos con derechos civiles lo demuestran ${ }^{35}$.

\footnotetext{
32 Ver nota 8.

33 En Chile, diríamos peras y manzanas.

${ }^{34}$ Estos comentarios no tienen por finalidad negar el hecho de que las obligaciones terciarias de los DESC sean vagas. Más bien, se trata de realzar que la imprecisión está dada por el tipo de obligación, no el tipo de derecho, y por tanto, ocurre tanto con los DCP como con los DESC. HUNT (19956, p. 34) agrega que, en cualquier caso, la legislatura puede determinar el nivel en el cual el Estado debe satisfacer sus obligaciones y, al hacerlo, puede contribuir a reducir el nivel de indeterminación de los deberes, por la vía de instituir regulación precisa.

35 Consideremos el derecho a la vida. Simplificando bastante las cosas, este derecho significa el derecho a que no me maten arbitrariamente (a nivel filosófico es discutible qué significa tener un deber de no matar.
} 
2.2. Un segundo comentario en contra de la objeción de vaguedad de los DESC está relacionado con la práctica constitucional. Unos autores (Scott \& Macklem, 1992, p. 72) señalan que la precisión de los derechos no es algo lingüístico ni tampoco algo que dependa de la naturaleza o esencia de los derechos sino de una práctica de interpretación, sostenida a través del tiempo. Esto es válido tanto para los DCP como para los DESC. Si podemos concordar -dicen estos autores- en que en cierto sentido los DESC pueden ser más imprecisos que los DCP, ello se debe precisamente a que la pretendida no justiciabilidad de esos derechos los ha dejado fuera de las cortes y, consecuencialmente, privados de una práctica continua de interpretación judicial. Por otra parte, si los DCP son precisos, ello es consecuencia de la práctica de la labor judicial ${ }^{36}$. Además, cabe destacar que los DCP también siguen evolucionando y su desarrollo no ha llegado

Ver WALDRON (1995) p. 577, citando a Nozick, Williams, Scheffler). ¿A qué tiene derecho el titular del derecho a la vida? Sin duda, tiene derecho a que el Estado no lo mate arbitrariamente. Esto correspondería a la obligación primaria de respetar el derecho a la vida. Pero, ¿a qué más tiene derecho el titular, si pensamos en el nivel secundario de proteger el derecho a la vida y en el nivel terciario de satisfacer el mismo derecho? ¿Tiene derecho a protección policial permanente, a que el Estado financie guardaespaldas para él, a que instale sistemas de alarmas y protección en su casa, etc? No cabe duda que la respuesta que cualquiera daría a estas hipótesis sería negativa. Por tanto, fente a la obligación precisa de que el Esado no lo mate arbitrariamente, el sujeto tiene además derecho a otras cosas, en virtud de las obligaciones secundarias y terciarias, pero no sabemos bien a qué. Quizá no podamos decir nada más preciso que indicar que las personas tienen derecho a que exista una fuerza policial de tamaño, entrenamiento y equipamiento razonables, que patrulle las calles y atienda llamados de emergencia; a que exista un sistema legal que contemple códigos que prohíban los crímenes, procedimientos legales para sancionarlos, y jueces que lo hagan, etc. Esto es bastante impreciso, porque hay muchas formas de hacer lo anterior. ¿Qué debe hacer la policía, exactamente, para prevenir crímenes contra la vida? ¿Cuán severos deben ser los Códigos Penales? ¿Qué tipos de leyes son disuasivas? ¿Cómo deben ser las políticas de persecución criminal? Existe algo claro sobre el derecho a la vida -que no me maten arbitrariamente- pero todo lo demás es bastante vago e impreciso, y corresponderá a la administración del Estado y al Poder Legilativo diseñar diversas políticas destinadas a cumplir con las obligaciones de proteger y satisfacer, que emanan del derecho a la vida.

Continuando con el derecho a la vida en su dimensión más básica, en el nivel primario, de todos modos existen muchas interrogantes: ¿prohíbe el aborto, la anticoncepción, la eutanasia, la pena de muerte? Estos asuntos son discutidos en el mundo hoy, en diversos países, y las cortes constitucionales a veces tienen serios problemas al intentar responder algunas de estas preguntas. Esto demuestra que un derecho civil, de libertad, clásico, como el derecho a la vida, no está libre de importantes imprecisiones y vaguedades.

Muchos otros derechos civiles tradicionales presentan problemas de precisión. Por ejemplo, igualdad ante la ley. ¿Se requieren programas de acción afirmativa?¿Qué modelos de igualdad deben ser escogidos en determinadas áreas? La prohibición del matrimonio gay y la adopción, ¿es discriminatoria? Etc. En materia de debido proceso, ¿qué se requiere para satisfacer el estándar de un tribunal independiente e imparcial? ¿Qué tipos de abogados debe proporcionar el Estado cuando presta asistencia jurídica de oficio? ¿Qué es necesario para asegurar un debido proceso? Etc.

36 SCOTT \& MACKLEM (1992) p. 72, lo señalan del siguiente modo: "[T]he key to making obligations attendant upon the constitutionalization of social rights clearer and more precise is to have experience with actual, real-life situations that call upon courts to move from the abstract to the concrete and develop the meaning and scope of social rights with some degree of precision (...) The specific shape and contour of a right is the result of years of repeated applications of practical reasoning to facts at hand. The lack of precision associated with many social rights should not be held up as a justification for their non-entrenchment. On the contrary, non-entrenchment is to a very large extent the reason for the lack of precision. Historical, ideological, and philosophical exclusions of social rights from adjudicative experience have resulted in a failure to accumulate experience that would render the imprecision of social rights less and less true as time goes on". 
a su fin. La práctica constitucional sigue proveyendo precisión a tales derechos y resolviendo diversos problemas que se van presentado 37 . En consecuencia, tanto los DCP como los DESC están sujetos a la práctica constitucional y a la interpretación, un proceso que incrementa la precisión de tales derechos a través del tiempo, independientemente de la mayor ventaja que en dicho proceso los DCP puedan exhibir comparados con los DESC.

Esta dinámica se aprecia también en la comunidad internacional. Se han dado pasos progresivos y exitosos hacia la precisión de los derechos contenidos en el PIDESC y se han formulado criterios para identificar las violaciones de parte de los Estados a los derechos recogidos en los tratados. Los Principios de Limburg, de 1987, constituyen un primer gran paso en esa dirección ${ }^{38}$, y diez años después el proceso continuó con las Directrices de Maastricht, que especifican claramente violaciones al PIDESC, sea por acción u omisión ${ }^{39}$. Ciertamente, los problemas de precisión no han desaparecido, pero

${ }^{37}$ Ejemplos son los casos del derecho a la vida, igualdad ante la ley, debido proceso, libertad de expresión, etc. Ver HaYsOM (1992) p. 457.

38 Por ejemplo, la sección B de tales principios enuncia 19 principios para interpretar y proveer precisión para el enfoque y extensión del artículo 2 (1) del PIDESC, y el principio 72 especifica situaciones muy claras de infracción de parte del Estado al convenio. "72. A State party will be in violation of the Covenant, inter alia, if: it fails to take a step which it is required to take by the Covenant; it fails to remove promptly obstacles which it is under a duty to remove to permit the immediate fulfilment of a right; it fails to implement without delay a right which it is required by the Covenant to provide immediately; it wilfully fails to meet a generally accepted international minimum standard of achievement, which is within its powers to meet; it applies a limitation to a right recognized in the Covenant other than in accordance with the Covenant; it deliberately retards or halts the progressive realization of a right, unless it is acting within a limitation permitted by the Covenant or it does so due to a lack of available resources or force majeure; it fails to submit reports as required under the Covenant".

39 "14. Violations of economic, social and cultural rights can occur through the direct action of States or other entities insufficiently regulated by States. Examples of such violations include: (a) The formal removal or suspension of legislation necessary for the continued enjoyment of an economic, social and cultural right that is currently enjoyed; (b) The active denial of such rights to particular individuals or groups, whether through legislated or enforced discrimination; (c) The active support for measures adopted by third parties which are inconsistent with economic, social and cultural rights; (d) The adoption of legislation or policies which are manifestly incompatible with preexisting legal obligations relating to these rights, unless it is done with the purpose and effect of increasing equality and improving the realization of economic, social and cultural rights for the most vulnerable groups; (e) The adoption of any deliberately retrogressive measure that reduces the extent to which any such right is guaranteed; ( $f$ ) The calculated obstruction of, or halt to, the progressive realization of a right protected by the Covenant, unless the State is acting within a limitation permitted by the Covenant or it does so due to a lack of available resources or force majeure; $(g)$ The reduction or diversion of specific public expenditure, when such reduction or diversion results in the non-enjoyment of such rights and is not accompanied by adequate measures to ensure minimum subsistence rights for everyone". "15. Violations of economic, social, cultural rights can also occur through the omission or failure of States to take necessary measures stemming from legal obligations. Examples of such violations include: (a) The failure to take appropriate steps as required under the Covenant; (b) The failure to reform or repeal legislation which is manifestly inconsistent with an obligation of the Covenant; (c) The failure to enforce legislation or put into effect policies designed to implement provisions of the Covenant; (d) The failure to regulate activities of individuals or groups so as to prevent them from violating economic, social and cultural rights; (e) The failure to utilize the maximum of available resources towards the full realization of the Covenant; $(f)$ The failure to monitor the realization of economic, social and cultural rights, including the development and application of criteria and indicators for assessing compliance; $(g)$ The failure to remove promptly-obstacles which it is under a duty to remove to permit 
los opositores a los DESC deben admitir los progresos que ha habido en el ámbito internacional. Un autor (Puta-Chekwe, 2001, pp. 48-49) está convencido de que los Principios de Limburg y las Directrices de Maastricht han contribuido al colapso de la dicotomía entre los DCP y los DESC; incluso, ha señalado que estas directrices han contribuido a disipar el mito de que los DESC sean derechos de diferente naturaleza que los DCP.

\section{EFECTIVIDAD INMEDIATA VERSUS PROGRESIVIDAD}

Este fue el tercer criterio para diferenciar los DCP de los DESC. El argumento sostiene que los derechos civiles clásicos son efectivos en términos inmediatos, en tanto los DESC son efectivos solo de manera progresiva. Por ello, el Estado no podría ser compelido - por ejemplo, a través de la judicatura- a hacerse cargo de los DESC, como sí de los DCP. Existen diversos comentarios en contra de este argumento:

3.1. Dicho argumento sería correcto a condición de que aceptáramos que los DCP solamente envuelven obligaciones negativas y abstención de parte del Estado, frente a los DESC que involucrarían solamente obligaciones positivas que suponen la intervención del Estado. Sin embargo, hemos visto que esto no es así. Los derechos civiles "negativos" comprenden obligaciones positivas que provienen de los niveles secundario y terciario, y los derechos económicos "positivos" contienen obligaciones negativas de abstención que dimanan del nivel primario de respetar. Siendo ese el caso, los derechos civiles tendrán niveles de obligaciones que solo pueden implementarse de manera progresiva, mientras que los DESC tendrán cierto tipo de obligaciones que pueden ser exigidas en lo inmediato. Por tanto, la distinción colapsa. En efecto, y como hemos visto, tanto los DCP como los DESC poseen tipos de obligaciones que son implementables tanto de manera inmediata como progresiva.

3.2. Se ha señalado que en relación con la práctica de la jurisdicción constitucional e internacional, los jueces han desarrollado un rol significativo respecto del control sobre el cumplimento progresivo de los derechos tradicionales ${ }^{40}$. Por ejemplo, en materia de debido proceso y equidad procedimental, las cortes han objetado demoras no

the immediate fulfilment of a right guaranteed by the Covenant; (b) The failure to implement without delay a right which it is required by the Covenant to provide immediately; (i) The failure to meet a generally accepted international minimum standard of achievement, which is within its powers to meet; (j) The failure of a State to take into account its international legal obligations in the field of economic, social and cultural rights when entering into bilateral or multilateral agreements with other States, international organizations or multinational corporations".

40 SCOTT у MACKLEM (1992) pp. 47-48, afirman: "[T] he negative-positive divide does not neatly distinguish sets of rights. Moreover, the fact that a right can entail a positive obligation does not mean that the judiciary is incapable of having something constructive to say about the scope and remedial requirements of that right. As a matter of practice, traditional civil and political rights have been found to entail positive rights at both the constitutional and international levels, either because of the explicit wording of the text or as a necessary implication of the right. The judiciary has demonstrated that it is prepared and able to require positive state action in the context of traditional civil and political rights". 
razonables respecto de acusados que esperan un juicio, y se ha declarado que los Estados deben incrementar la capacidad del sistema judicial, construyendo nuevas cortes y contratando más jueces y personal administrativo (Scott \& Macklem, 1992, p. 47, 50-51). Asimismo, la extensión de algunos derechos procedimentales en procedimientos administrativos ha incrementado considerablemente los costos de tales tribunales. La garantía del derecho a un defensor, tradicionalmente considerado un derecho civil, ha resultado en que las cortes ordenen al Estado asumir los gastos que ello implica ${ }^{41}$.

El propósito de estos ejemplos no es mostrar que los derechos civiles tienen exactamente los mismos problemas de implementación progresiva que los DESC, sino probar que los DCP tiene problemas de implementación debido a las obligaciones positivas que dimanan de ellos, las que no pueden ser cumplidas de inmediato (Hunt, 1993, p. 157).

3.3. Un tercer comentario, mencionado antes, es que no todos los deberes involucrados en los DESC, tal como han sido reconocidos por el PIDESC, son deberes progresivos; algunos de ellos son efectivos de inmediato, tal como los han reconocido el Comentario General No 3 , del Comité DESC del PIDESC, en la sección $1^{42}$, y el Principio de Limburg No $8^{43}$. Un claro ejemplo corresponde a las exigencias de no

\footnotetext{
${ }^{41}$ En materia de derecho a la vida, libertad y seguridad personal, SCOTT \& MACKLEM (1992) p. 53) estos autores señalan que "...the duty to protect is so important that states are required to give a certain priority of resources to police and related means of protection as the court system".

El caso citado es Velásquez Rodríguez, que involucró la desaparición de personas a manos de oficiales el Estado en Honduras. La Corte Interamericana de Justicia consideró que Honduras violó la Convención Americana por su negligencia de no haber investigado las desapariciones. También mencionan estos autores casos que involucran la libertad de expresión y de reunión, en que la policía falló en proteger a quienes se manifestaban (SCOTT y MACKLEM, 1992, pp. 55-62). En materia de derecho a la privacidad y la vida familiar, se cita el caso Marckx, del sistema europeo, en que el Estado fue condenado por haber fallado en proteger a la familia no tradicional. Además, la corte declaró que el respeto por la vida familiar implica en particular la existencia de legislación doméstica que establezca salvaguardias que hagan posible la integración del niño a su familia desde el momento del nacimiento. Otro caso de la Corte Europea es el caso X e Y, donde la ley holandesa falló en disponer la persecución criminal de una persona que había cometido asalto sexual en contra de una niña con discapacidad mental. "( $t)$ he law neither allowed her to press charges on her own nor provided for any guardian to so on her behalf". (Se citan otros casos de privacidad). En materia de derechos de los prisioneros, estos autores señalan: "Courts in the United States have long found there to be positive obligations on authorities for the conditions in prisons an other state institutions, such as those for mentally insane. Courts have ordered that prisons be built to relieve overcrowding and that committees be established to carry out programs of institutional reform laid down by the courts. Prison officials have been found to have engaged in cruel and unusual punishment, contrary to the Eight Amendment, after demonstrating "deliberate indifference" to a prisoner's medical needs. The constitutional right to access the courts has been interpreted as requiring prison officials to furnish prisoners with the tools necessary to research legal claims. To name but a few positive initiatives, courts have ordered an increase in custodial personnel, the construction of a prison hospital, and the provision of vocational training".

42 "II]n particular, while the Covenant provides for progressive realization and acknowledges the constraints due to the limits of available resources, it also imposes various obligations which are of immediate effect".

43 "Although the full realization of the rights recognized in the Covenant is to be attained progressively, the application of some rights can be made justiciable immediately while other rights can become justiciable over time".
} 
discriminación que aparecen establecidas en los Principios de Limburg Nos $22^{44}, 37^{45}$ y $49^{46}$. Otro ejemplo es la obligación positiva de adoptar pasos hacia la realización progresiva de los derechos reconocida en el PIDESC, la que es efectiva de inmediato, tal como lo ha señalado el Comité en su Comentario General No. 3 sección $2^{47}$. Esta exigencia ha sido recogida también por los Principios de Limburg ${ }^{48}$. La razón de estas prescripciones es que no existen restricciones presupuestarias ni consideraciones basadas en la escasez de recursos que impidan al Estado diseñar y comenzar a implementar planes con el propósito de asumir el cumplimiento de su deber de proporcionar una implementación progresiva a los DESC, según sus propias capacidades financieras y de gestión ${ }^{49}$. Salvo casos rarísimos, debería ser difícil para un Estado alegar que se encuentra total y completamente impedido de satisfacer un DESC en ningún nivel posible.

3.4. Finalmente, se puede señalar que todas las obligaciones negativas que envuelven los DESC (nivel primario, respetar) son efectivas de inmediato, precisamente por su carácter negativo, y no de manera progresiva (Brems, 2001, p. 447; Eide, 1989, pp. $40-$ 41; Eide et al., 1995, p. 38).

\section{OTROS ARGUMENTOS}

En la primera sección se explicó que había otros criterios para distinguir los DCP de los DESC, aunque probablemente eran de menor envergadura e importancia. En esta parte, se abordan y comentan de manera breve.

\subsection{Base ideológica}

Según este criterio, los DESC son a menudo percibidos como profundamente ideológicos, que requieren una inaceptable intervención del Estado en asuntos domésti-

\footnotetext{
44 22. "Some obligations under the Covenant require immediate implementation in full by all States parties, such as the prohibition of discrimination in article 2(2) of the Covenant".

45 37. "Upon becoming a party to the Covenant States shall eliminate de jure discrimination by abolishing without delay any discriminatory laws, regulations and practices (including acts of omission as well as commission) affecting the enjoyment of economic, social and cultural rights".

46 49. "Laws imposing limitations on the exercise of economic, social and cultural rights shall not be arbitrary or unreasonable or discriminatory".

47 "The other is the undertaking in article 2 (1) "to take steps", which in itself, is not qualified or limited by other considerations".

48 "16. All States parties have an obligation to begin immediately to take steps towards full realization of the rights contained in the Covenant".

${ }^{49}$ Continuando con el Comentario General No 3, la sección 5 establece: "II]n addition, there are a number of other provisions in the International Covenant on Economic, Social and Cultural Rights, including articles 3, 7 (a) (i), 8, 10 (3), 13 (2) (a), (3) and (4) and 15 (3) which would seem to be capable of immediate application by judicial and other organs in many national legal systems". El comité DESC de la ONU ha declarado que un mínimo de realización de los DESC puede ser efectivo de inmediato. Al efecto, el Comentario General № 3 , sección 10 señala: "On the basis of the extensive experience gained by the Committee, as well as by the body that preceded it, over a period of more than a decade of examining States parties' reports the Committee is of the view that a minimum core obligation to ensure the satisfaction of, at the very least, minimum essential levels of each of the rights is incumbent upon every State party. (...) If the Covenant were to be read in such a way as not to establish such a minimum core obligation, it would be largely deprived of its raison d'être".
} 
cos, o son inherentemente incompatibles con una economía de libre mercado, en tanto los DCP son no ideológicos por naturaleza y son potencialmente compatibles son la mayoría de los regímenes políticos.

La respuesta a ese argumento consiste en cuestionar la suposición en que se basa: que sea posible que ciertos derechos sean neutrales y que la teoría que subyace a ellos lo sea también. Tal suposición es inaceptable: una teoría de los derechos humanos “...comprende una teoría de la justicia (aunque sea parcial o incompleta); envuelve una forma de abordar las obligaciones y deberes que corresponden a los derechos reclamados, y presupone una concepción de las necesidades humanas y del desarrollo humano" (Beetham, 1995, p. 45) ${ }^{50}$. En suma, todo derecho es ideológico.

\subsection{Ausencia de compromiso}

Según este argumento, los DCP están relacionados con valores compartidos, aquellos con los cuales los gobiernos en verdad se encuentran comprometidos, y generan problemas que los gobiernos pueden manejar. En cambio, los DESC no han obtenido igual grado de compromiso de parte de los gobiernos precisamente porque involucran problemas que, en general, o son inmanejables o difícilmente manejables.

La respuesta a este argumento es que, en verdad, no se trata de un argumento en contra de los DESC. Lo que se dice, aunque sea cierto, corresponde a una afirmación de hecho. Como tal, no constituye una guía de acción acerca de qué se debe hacer con los DESC. Por otra parte, lo que se dice de ellos ocurre también con los DCP en ciertos países. En efecto, en vastas áreas del planeta existen regímenes que tienen poca consideración con los derechos civiles. Pero ante esa realidad no creemos que los derechos civiles deban ser dejados de lado o no deban ser justiciables. Al contrario, mientras menos se respeten, el argumento en su favor se hace más urgente y necesario. Además, el compromiso de diversos países y de la comunidad internacional con los DESC ha ido creciendo en las últimas décadas. A nivel internacional vemos esfuerzos notables de proveer mayor precisión a estos derechos, de identificar formas de violación y de sugerir remedios para las mismas, como los Principios de Limburg, las Directrices de Maastricht y los Comentarios Generales del Comité DESC del PIDESC. Fuera de eso, tenemos muchos ejemplos de jurisdicciones domésticas ${ }^{51}$ e internacionales ${ }^{52}$ que han reconocido formas de hacer justiciables los DESC. Esos ejemplos muestran un compromiso no menor de parte de algunos Estados con tales derechos y su justiciabilidad. En consecuencia, el argumento que se analiza no parece ser especialmente persuasivo.

\subsection{Autonomía versus incapacidad}

Como vimos en la sección I, Kelley ha presentado un argumento filosófico en contra de los DESC, basado en cierta concepción de los seres humanos. El señaló que los derechos de libertad dejan a los individuos responsables de vivir sus propias vidas y

\footnotetext{
50 Traducción libre del autor.

51 Como la Corte Constitucional Sudafricana, o la Corte Suprema de la India.

${ }^{52}$ Como la Corte Europea de DD.HH.
} 
satisfacer sus propias necesidades, y proporcionan la libertad necesaria para afrontar sus responsabilidades. En cambio, los derechos de bienestar no permiten esto; reflejan una visión colectivista o comunitaria que sacrifica la libertad y la autonomía. Diversos comentarios se pueden esgrimir en contra de esta postura:

1. Asumiendo que estas concepciones han sido correctamente retratadas por Kelley, podemos controvertir que la concepción que ensalza Kelley sea objetivamente preferible. Si nos viéramos forzados a elegir entre ambas, bajo condiciones imparciales, como cubiertos hipotéticamente por un velo de ignorancia ralwsiano, sería dudoso que los individuos inevitablemente prefirieran la concepción individualista de Kelley. La razón es que si el individuo actúa racionalmente, probablemente evitaría elegir una condición o un sistema de derechos que lo deje, a perpetuidad, desprotegido si es que tiene la mala suerte de nacer y vivir en los lugares más desaventajados de la estructura social. De hecho, el segundo principio de Rawls se funda precisamente en esta idea.

2. La perspectiva de Kelley parece ignorar la evolución que cualquier persona debería poder apreciar en el mundo hoy, que consiste precisamente en que de manera progresiva se están tomando cada vez más en serio los DESC. Siendo ese el caso, ¿por qué debería ser obvio, como pretende Kelley, que una concepción que él llama libertaria sería preferible a la que él denomina comunitaria?

3. Kelley presenta una concepción de la autonomía que prescinde de niveles mínimos de bienestar. Eso también es cuestionable, porque sin ciertas necesidades básicas satisfechas, el individuo no puede ser un agente, ni siquiera puede elaborar una concepción de sí mismo como un ser autónomo. Kelley parece sostener que la libertad negativa es intrínsecamente valiosa, con independencia de las condiciones de vida material del individuo. No es necesario haber leído los manuscritos económico-filosóficos para entender que las condiciones de vida material en algo deben condicionar los niveles de conciencia. Por ello, un cierto nivel de condiciones materiales de subsistencia es una condición sinne qua non para pensar siquiera en libertad negativa ${ }^{53}$.

4. Plant (2003, pp. 3-4) cree que es dudoso que la libertad negativa pueda ser categóricamente distinguida de las habilidades, capacidades, recursos y oportuni-

\footnotetext{
53 KELlER (2003) pp. 592-593 presenta un argumento a favor de los DESC basado en la idea de persecución de la felicidad, reconocida por la Declaración de Independencia de los EE.UU. Su argumento es el siguiente: "...the right to pursue happiness does not require the government to provide happiness, "but the assistance of others - including the government - is nevertheless sometimes required, because without the minimum necessities of life an individual is forced to struggle for daily survival and, therefore, has no opportunity to pursue his or her happiness". "What organized societies and instituted governments can do is provide human beings with the external conditions indispensable to the pursuit of happiness, facilitating but not ensuring its attainment. The right to pursue happiness is, therefore, a right to these indispensable external conditions...". These indispensable external conditions may encompass a broad range of rights. In short, they include "a right to a decent livelihood," and the "comforts and conveniences of life that are accessory to a successful pursuit of happiness". They include the right "to basic education, health care, food, housing, and clothing, for without these things an individual is denied an opportunity to pursue a good and happy life"'.
} 
dades. Kelley distingue entre la libertad y el valor de la libertad. Sin embargo, esa distinción no tiene sentido. Si separamos ambos cosas -arguye Plant- entonces el valor de la libertad para los seres humanos sería difícil de explicar ${ }^{54}$. Agrega que estar libre de coerción carece de valor para las piedras o un atado de pasto, pero sí lo tiene para los seres humanos "...porque son capaces de realizar opciones, establecer objetivos, esto es, actuar como agentes" 55 . "[L]a libertad solo puede ser inteligible en términos de una concepción de la agencia humana (...) la que no puede ser formulada con prescindencia de una referencia a las habilidades, necesidades y capacidades humanas" 56 .

\subsection{Pobreza}

Esta objeción señalaba que los DESC no son remediables -y, por ello, no justiciables- porque no existe un agente causal de la pobreza. Este punto es muy relevante para países pobres o en vías de desarrollo, como Chile. Existen varias respuestas a este argumento:

1. Esta objeción falla al no distinguir violaciones a los derechos contenidos en los tratados de parte de los Estados, de una situación de pobreza que haga imposible para el Estado cumplir con sus deberes económicos, impuestos por el tratado.

54 “[P]or qué querría yo estar libre de coerción? Seguramente, la respuesta sería que si estoy libre de ella entonces tendré la capacidad de vivir mi vida forjada por mis propias intenciones, objetivos y propósitos. Por eso la libertad es valiosa para mí. Luego, si el valor de la libertad es explicado en términos de lo que yo soy capaz de hacer con ella, entonces se torna bastante difícil de decir que la libertad y la habilidad son totalmente separables". (PLANT, 2003, p. 3). Traducción libre del autor.

55 Traducción libre del autor.

56 Traducción libre del autor.'PLANT (2003) pp. 5-7 está consciente de que este argumento está lejos de justificar los DESC, pero tiene el mérito de socavar la idea de que la libertad y la habilidad son cosas radicalmente diferentes, un punto que fue invocado por Hayek en The Constitution of Liberty. Según este autor, la inhabilidad y la libertad no se encuentran conectadas: “[E]l ámbito de posibilidades físicas al interior del cual una persona puede escoger no tiene relevancia directa para la libertad. El escalador de roca que se encuentra en un paso difícil y que ve solo una vía para salvar su vida es incuestionablemente libre, aunque difícilmente diríamos que tiene alternativas". Plant no comparte esta visión. El señala que otra forma de ilustrar la importancia de la agencia humana para comprender la libertad es por la vía de reconocer que una “...habilidad genérica de realizar X es una condición necesaria para poder determinar si A es o no libre para ejecutar X”. También menciona un argumento de Charles Taylor, según el cual si nos centramos solamente en la libertad negativa, entonces debeíamos aceptar que un país con menos reglas es más libre que uno con más reglas. Pero, un país con más reglas, que permite a los ciudadanos emigrar y criticar al gobierno, es más libre que tiene menor reglas pero lo torna imposible. Descansando ahora en Cohen -quien presenta un ejemplo para argumentar que la falta de dinero se transforma en una restricción para la libertad negativa- Plant presenta el siguiente ejemplo: Si un país totalitario prohíbe legalmente viajar, mi libertad se ve restringida. Esa restricción proviene de la ley. Ahora, si viajar no está restringido por la ley pero yo no tengo el dinero para pagar el pasaje, no voy a poder viajar porque la ley prohíbe viajar sin un ticket. En consecuencia, en ambas situaciones el efecto es el mismo. Las inhabilidades económicas al interior de cierto esquema legal impactan en la libertad de manera similar a restricciones legales. La consecuencia de este argumento es: si usted necesita recursos para hacer X pero carece de ellos, entonces usted no es libre para ejecutar X. Esta conclusión muestra la conexión entre libertad y capacidad y proporciona una objeción a la línea de razonamiento en contra de los DESC examinada en esta parte. 
Ambas situaciones deben ser distinguidas porque la primera hace responsable al Estado por su incumplimiento sea en virtud de acciones u omisiones, mientras que la segunda no. Lo que se necesita es poder identificar qué actos u omisiones que socavan los DESC son imputables al Estado y cuáles no. Esto es necesario si deseamos supervisar el cumplimiento de las obligaciones impuestas por los tratados (Melish, 2002, pp. 39-40) lo que es indispensable para intentar mejorar la causa de los DESC. Puesto en otras palabras, la existencia de la pobreza no torna en irrelevante el PIDESC ni posterga su supervisión hasta que ella desaparezca. Esta idea aparece claramente reconocida en las Directrices de Maastricht ${ }^{57}$.

2. Violaciones y deterioro de los DESC no ocurren solamente en lugares donde existe pobreza. Además, los DESC pertenecen a todas las personas, pobres, de clase media y ricas, y las obligaciones de los tratados de respetarlas, protegerlas y satisfacerlas son transversales. En consecuencia, el cumplimiento del tratado puede ser supervisado independientemente del nivel de riqueza o pobreza de la sociedad de que se trate ((Melish, 2002, p. 40).

3. Los países pobres no tienen una obligación menor respecto del PIDESC que los países ricos, pues la obligación de implementar esos derechos progresivamente está condicionada obviamente por los recursos económicos disponibles en cada Estado. Ciertamente la implementación máxima es obligatoria, pero ese máximo es diferente para diversos Estados. Esto ha sido reconocido tanto por los Principios de Limburg ${ }^{58}$ como por las Directrices de Maastricht ${ }^{59}$.

57 "13. In determining which actions or omissions amount to a violation of an economic, social or cultural right, it is important to distinguish the inability from the unwillingness of a State to comply with its treaty obligations. A State claiming that it is unable to carry out its obligation for reasons beyond its control has the burden of proving that this is the case. A temporary closure of an educational institution due to an earthquake, for instance, would be a circumstance beyond the control of the State, while the elimination of a social security scheme without an adequate replacement programme could be an example of unwillingness by the State to fulfil its obligations".

58 "23. The obligation of progressive achievement exists independently of the increase in resources; it requires effective use of resources available".

"25. States parties are obligated, regardless of the level of economic development, to ensure respect for minimum subsistence rights for all”.

"27. In determining whether adequate measures have been taken for the realization of the rights recognized in the Covenant attention shall be paid to equitable and effective use of and access to the available resources".

59 "9. Violations of the Covenant occur when a State fails to satisfy what the Committee on Economic, Social and Cultural Rights has referred to as "a minimum core obligation to ensure the satisfaction of, at the very least, minimum essential levels of each of the rights [...]. Thus, for example, a State party in which any significant number of individuals is deprived of essential foodstuffs, of essential primary health care, of basic shelter and housing, or of the most basic forms of education is, prima facie, violating the Covenant. Such minimum core obligations apply irrespective of the availability of resources of the country concerned or any other factors and difficulties".

"10. In many cases, compliance with such obligations may be under-taken by most States with relative ease, and without significant resource implications. In other cases, however, full realization of the rights may depend upon the availability of adequate financial and material resources. Nonetheless, as established by Limburg Principles 25-28, and confirmed by the developing jurisprudence of the Committee on Economic, Social and Cultural Rights, resource scarcity does not relieve States of certain minimum obligations in respect of the implementation of economic, social and cultural rights". 
4. Plant (2003, p. 14) presenta otro argumento. Los críticos de los DESC señalan que no es posible asumir una responsabilidad colectiva por consecuencias indeseadas - como las derivadas de crisis en los mercados- y conferir a los individuos DESC para protegerse de tales consecuencias; la pobreza no genera derechos. Plant responde señalando que si bien las consecuencias negativas de fallas del mercado no son deseadas, son -sin embargo- previsibles y este grado de previsibilidad funda la idea de responsabilidad colectiva ${ }^{60}$. Los DESC, financiados por impuestos, podrían ser la manera como asumimos responsabilidad social por las consecuencias no queridas pero previsibles del mercado, como la pobreza $^{61}$.

\section{CONCLUSIONES}

1. Se han propuesto diferentes criterios para distinguir entre los DCP y los DESC. Algunos son más conocidos que otros, y también varían en importancia. La distinción más conocida parece ser entre derechos negativos/de abstención/sin costo y derechos positivos/de intervención/costosos. Dicha distinción es incorrecta considerando que todo derecho envuelve tres niveles de obligaciones. Algunas son positivas, requieren intervención del Estado e implican costos para el mismo, mientras que otras no.

2. Otra distinción importante es aquella referida a la precisión o imprecisión de las obligaciones que emanan de los derechos. Sin embargo, hemos visto que todo derecho implica obligaciones primarias, secundarias y terciarias, siendo las primeras precisas y las restantes imprecisas, de modo que todo derecho es preciso e impreciso a la vez, dependiendo de la obligación de que se trate.

3. Una tercera distinción importante es aquella referida a la implementación inmediata o progresiva de los derechos. Sobre esto, vale la misma consideración señalada en el número anterior, pues la implementación depende de la obligación involucrada.

4. En consecuencia, si bien existen algunas diferencias entre los DCP y los DESC, ellas claramente han sido exageradas y no justifican una oposición cerrada y categórica a la justiciabilidad de los DESC.

5. Finalmente, si bien se advierten ciertos problemas para hacer justiciables algunas obligaciones (como las secundarias o las terciarias), eso ocurre tanto con los DESC como con los DCP. Por tanto, no justifica una crítica exclusiva para los DESC.

\footnotetext{
60 "A nivel de responsabilidad individual, tanto moral como jurídica, podemos ser hechos moral y legalmente responsables por la razonable previsibilidad de las consecuencias de nuestras acciones, aunque no sean deseadas". (PLANT, 2003, p. 14) Traducción libre del autor.

${ }^{61}$ Esta consideración dirige nuestra atención al problema de quién tiene la responsabilidad. Hablar de responsabilidad social no proporciona identificación del poseedor de dicha responsabilidad. La respuesta que Plant (2003) p. 15, ofrece es que nadie es directamente responsable de satisfacer las necesidades de los demás, sino únicamente de contribuir con un sistema tributario.
} 


\section{BIBLIOGRAFÍA CITADA}

Abramovich, Víctor y COURTIS, Christian (2002): Los derechos sociales como derechos exigibles (Madrid, Editorial Trotta) 254 pp.

AdDO, Michael K (1988): "The Justiciability of Economic, Social and Cultural Rights", Commonwealth Law Bulletin, vol. 14: pp. 1425-1432.

Alston, Philip (1981-1982): "A Third Generation of Solidarity Rights: Progressive Development or Obfuscation of International Human Rights Law?", Netherlands International Law Review, vol. 28-29, pp. 307-322.

Alston, Philip and Quinn, Gerard (1987): “The Nature and Scope of States Parties' Obligations under the International Covenant on Economic, Social and Cultural Rights”, Human Rights Quarterly, vol. 9: pp. 157-229.

Alston, Philip (1997): "Making Economic and Social Rights Count: A strategy for the Future", The Political Quarterly: pp. 188-195.

Andreassen, Bard-Anders; Smith, Alan G.; Stokke, Hugo (1992): "Compliance with Economic and Social Human Rights: Realistic Evaluations and Monitoring in the Light of Immediate Obligations”, en: EIDE Absjorn \& HAGTVET Berut (edit.), Human Rights in Perspective: A Global Assessment (Oxford, Blackwell Publishers) pp. 252-267.

Beetham, David (1995): "What future for Economic and Social Rights?", Political Studies, vol. 43: pp. 41-60.

BERLIn, Isaiah (1969): Two Concepts of Liberty, en BERLIN, Isaiah: Four Essays on Liberty (Oxford, Oxford University Press) 206 pp.

Brems, Eva (2001): Human Rights: Universality and Diversity (The Hague, Martinus Nijhoff Publishers) 574 pp.

Cranston, Maurice (1967): "Human Rights, Real and Supposed", en: RAPHAEL, D.D. (edit.), Political Theory and the Rights of Man (Indiana, Indiana University Press) pp. 43-94.

Craven, Matthew C.R (1993): “The Domestic Application of the International Covenant on Economic, Social and Cultural rights", Netherlands Internacional Law Review, vol. XL: pp. 367-404.

De Villiers, Bertus (1995): "Social and Economic Rights", en: VAN DYK, David; Dugard, John; De Villiers, Bertus; Davis; Dennis (edit.), Rights and Constitutionalism (Oxford, Clarendon Press) 599-628.

EIDE, Asbjorn (1989): "Realization of Social and Economic Rights and the Minimum Threshold Approach”, Human Rights Law Journal, vol. 10: pp. 35-51.

EIDE, Absjorn (1992): "National Sovereignty and International Efforts to Realize Human Rights", en: ABSJORN Eide \& BERUT Hagtvet (edit.) Human Rights in Perspective: A Global Assessment (Oxford, Blackwell Publishers) pp. 3-30.

EIDE, Absjorn \& ROSAS, Allan (1995): "Economic, Social and Cultural Rights: a Challenge", en: EIDE Absjorn, KRAUSE Catarina \& ROSAS Allan (edit.), Economic, Social and Cultural Rights. A Textbook (Dordrecht 1, Martinus Nijhoff) pp. 15-40.

FrIED, Charles (1978): Right and Wrong (Cambridge, Harvard University Press) 226 pp. 
Figueroa García-Huidobro, Rodolfo " "Justificación del rol de las cortes haciendo justiciables los derechos..."

GÓmeZ, Mario (1995): “Social Economic Rights and Human Rights Commissions”, en: Human Rights Quarterly, vol. 17: pp. 155-169.

GOODWIN-Gill, Guy S. (1984): "Obligations of Conduct and Result", en: AlSTON, P

y Tomasevski, K. (edit.), The Right to Food (Boston, M. Nihoff) pp. 111-118.

HaYsom, Nicholas (1992): "Constitutionalism, Majoritarian Democracy and Socio-

Economic Rights", South African Journal on Human Rights, vol. 8: pp. 451-463.

Hill, Dilys M (1992): "Rights and their realization", en: BEDDARD, Ralph \& Hill.

Dilys M. (edit.), Economic, Social and Cultural Rights (N.Y. St. Martin Press) pp. $1-19$.

HiRSCHL, Ran (2000): “Negative' Rights vs. 'Positive' Entitlements: a Comparative Study of Judicial Interpretation of Rights in an emerging Neo-liberal Economic Order", Human Rights Quarterly, vol. 22: pp. 1060-1087.

Holmes, Stephen and Sunstein, Cass (1999): The Cost of Rights (New York, London

W.W. Norton \& Company) 255 pp.

HunT, Paul (1993): "Reclaiming Economic, Social and Cultural Rights", Waikato Law Review, vol. 1: pp. 141-163.

HunT, Paul (1996): Reclaming Social Rights. International and Comparative Perspectives (USA, Dartmounth Publishing Company) 313 pp.

JACKMAN, Martha (1992): "Constitutional Rethoric and Social Justice: Reflections on the Justiciability Debate”, en: BAKAn, Joel \& SCHneIDERMAn, David (edit.), Social Justice and the Constitution: Perspectives on a Social Union for Canada (Ottawa, Carleton University Press) pp. 17-94.

Keller, Linda M (2003): “The American Rejection Of Economic Rights As Human Rights \& The Declaration Of Independence: Does The Pursuit Of Happiness Require Basic Economic Rights?”, New York Law School Journal of Human Rights, vol. 19: pp. 557-613.

KELley, David (1998): A Life of One's Own (Washington, Cato Institute) 176 pp.

KOCH, Ida Elisabeth (2002): "Social Rights as Components in the Civil Right to Personal Liberty: Another Step Forward in the Integrated Human Rights Approach?”, en: Netherlands Quarterly of Human Rights, vol. 20, No 1: pp. 29-51.

Melish, Tara (2002): Protecting Economic, Social and Cultural Rights in the Inter-American Human Rights System. A Manual on Presenting Claims (New Haven, Orville H. Schell Jr. Center for International Human Rights, Yale Law School; Ecuador, Centro de Derechos Económicos y Sociales) 473 pp.

MUREINIK, Etienne (1992): "Beyond a Charter of Luxuries: Economic Rights in the Constitution", South African Journal on Human Rights, vol. 8: pp. 464-474.

PeCES-BARbA, Gregorio (1981): "Reflections on Economic, Social and Cultural Rights", Human Rights Law Journal, vol. 2: pp. 281-294.

PellonpäÄ, Matti (1993): Economic, Social and Cultural Rights, en: MACDONALD R. St., Matscher, F. \& Petzold, H. (edit.), The European System for the Protection of Human Rights (Dordrecht, Martinus Nijhoff Publishers) pp. 855-874.

Plant, Raymond (2003): "Social and Economic Rights Revisited", The King's College Law Journal, vol. 14: pp. 1-20. 
PuTA-CheKWE, Chisanga and Flood, Nora (2001): "From Division to Integration: Economic, Social and Cultural Rights as Basic Human Rights", en: MERALI, Isfahan y Oosterveld, Valerie (edit.), Giving Meaning to Economic, Social and Cultural Rights (Philadelphia, University of Pennsylvania Press) pp. 39-51.

SCOTT, Craig (1989): "The Interdependence and Permeability of Human Rights Norms: Towards a Partial Fusion of the International Covenants on Human Rights", Osgoode Hall Law Journal, vol. 27: pp. 769-879.

SCOTT, Craig y MACKLEM, Patrick (1992): "Constitutional Ropes of Sand or Justiciable Guarantees? Social Rights in a New South African Constitution”, University of Pennsylvania Law Review, vol. 141: pp. 1-148.

Shue, Henry (1980): Basic Rights. Subsistence, Affluence and U.S. Foreign Policy (Princeton University Press) 236 pp.

Shue, Henry (1984): “The Interdependece of Duties", en: Alston, P. y TOMASEvski, K. (edit.), The Right to Food (Boston, M. Nihoff) pp. 83-95.

Sunstein, Cass (1993): "Against Positive Rights", East European Constitutional Review, vol. 1-3 (1992-1994) pp. 35-38.

TÜRK, Danilo (1992): “The Realization of Economic, Social and Cultural Rights". Special Rapporteur. E/CN.4/Sub.2/1992/16.

VAN Hoof, G.J.H. (1984): “The Legal Nature of Economic, Social and Cultural Rights: a Rebuttal of Some Traditional Views", en: Alston, P. y TOMasevski, K. (edit.), The Right to Food (Boston, M. Nihoff) pp. 97-110.

VIERDAG, E.W. (1978): “The Legal Nature of the Rights Granted by the International Covenant on Economic, Social and Cultural Rights", Netherlands Yearbook of International Law, vol. IX: pp. 69-105.

Waldron, Jeremy (1995): “Rights”, en: Goodin, Robert E \& PeTtit, Philip (edit.), A Companion to Contemporary Political Philosophy (Cambridge, Blackwell) pp. 575-583.

\section{DOCUMENTOS INTERNACIONALES}

General Comments of the Committee on Economic, Social and Cultural Rights on the International Covenant on Economic, Social and Cultural Rights. HRI/GEN/1/Rev. 9 (Vol. I) Disponible en: <http://www2.ohchr.org/english/bodies/cescr/ comments.htm>

General Comments of the Human Rights Committee on the International Covenant on Civil and Political Rights. HRI/GEN/1/Rev. 9 (Vol. I). Disponible en: <http:// www2.ohchr.org/english/bodies/hrc/comments>

Limburg Principles on the Implementation of the International Covenant on Economic, Social and Cultural Rights. U.N. Doc. E/CN.4/1987/17, Annex.

Maastricht Guidelines on Violations of Economic, Social and Cultural Rights, Maastricht, January 22-26, 1997.

Vienna Declaration and Programme of Action. A/CONF.157/23. 12 de julio 1993. 